\title{
Intoxication volontaire à la doxylamine
}

\section{Self-poisoning by doxylamine}

\section{Sarah VAUTIER, Laurent HAVARD, Jean-Pierre DUPEYRON, François CHAST*}

Laboratoire de Toxicologie, Service de Pharmacie, Hôtel-Dieu, 1, place du Parvis Notre-Dame 75181 PARIS Cedex 04

* Auteur à qui adresser la correspondance : Dr François CHAST, Laboratoire de Toxicologie, Service de Pharmacie, Hôtel-Dieu, 1, place du Parvis Notre-Dame - 75181 PARIS Cedex 04

(Reçu le 4 novembre 2002 ; accepté le 9 décembre 2002)

\section{RÉSUMÉ}

Une jeune fille de 15 ans est admise aux urgences pour troubles du comportement, agitation et hallucinations, altérations des fonctions cognitives, troubles neurologiques et manifestations anticholinergiques importantes. L'examen psychiatrique ayant éliminé une cause psychotique, les médecins s'orientent vers une intoxication médicamenteuse, d'autant que les signes biologiques font craindre une rhabdomyolyse. Cette patiente a ingéré une pâte blanche qui sera analysée au laboratoire. Un prélèvement urinaire fera également l'objet d'un examen toxicologique.

L'analyse par chromatographie liquide haute performance couplée à un détecteur à barrette de diodes montre un pic isolé retrouvé à la fois dans les urines et la solution de poudre d'origine inconnue : temps de rétention et spectres $U V$ identiques, permettant de conclure à la présence de doxylamine, médicament antihistaminique essentiellement utilisé pour ses propriétés sédatives.

La patiente quitte l'hôpital après 6 jours de traitement symptomatique sans complications ni séquelles neurologiques. Cette intoxication médicamenteuse sévère liée à l'absorption d'une quantité importante de Donormyl®, spécialité en vente libre, doit éveiller l'attention des pharmaciens quant à sa dispensation à des patients tentés par un éventuel usage détourné.

\section{MOTS-CLÉS}

Anticholinergiques, Doxylamine, HPLC-BD, Intoxication médicamenteuse, Rhabdomyolyse.

\section{SUMMARY}

A 15-year old girl is admitted to emergency unit for behavior troubles, restlessness and hallucinations, cognitive, neurological and anticholinergic disturbances. Psychiatric examination being discordant with psychosis, physicians turn toward drug overdose. Patient's mother brought to the hospital a beaker, found in the girl's bedroom, containing a humid powder that looked as white paste. Part of this paste and a urine sample were analyzed. Immunochemical tests for drugs of abuse on urine sample and on an aqueous solution made with the paste were both negative.

After hydrolysis and solid-liquid extraction, urine and aqueous solution were analyzed by high performance liquid chromatography with diode array detection. A high absorbance peak was founded in the urine as well in the aqueous solution, with homologous UV spectrum. The comparison with spectrum library allowed to conclude for presence of doxylamine both in urine and in paste.

The young patient said she had bought several Donormy/TM boxes, without more explanations. This serious intoxication, with a low price OTC drug, may prompt pharmacists to a careful attention when delivering the drug to people at risk of misuse.

\section{KEY-WORDS}

Doxylamine, HPLC-DAD, Anticholinergic drugs, Rhabdomyolysis, Self-poisoning. 


\section{Introduction}

Certains médicaments, dont la toxicité est habituellement modeste, sont rarement utilisés dans les circonstances d'une intoxication volontaire. C'est la raison pour laquelle leur identification peut poser divers problèmes au moment de l'analyse toxicologique. Après les premiers tests d'orientation diagnostique, il peut être utile d'utiliser une méthode chromatographique, couplée à une identification précise au moyen d'un détecteur à barrette de diodes (CLHP-BD) ou d'un spectromètre de masse (CG-SM).

Une jeune fille âgée de 15 ans, est admise au service des urgences pour troubles du comportement, hallucinations, agitation et état confusionnel avec désorientation spatio-temporelle. Nous avons pu réaliser l'identification du toxique qu'elle a ingéré en utilisant une procédure de type HPLC-BD.

Lors de son admission, son état ne semble pas alarmant. Sa situation est considérée comme une «urgence relative». L'interrogatoire se révèle peu informatif car les propos de la mère et de la fille ne concordent pas.

L'examen clinique révèle :

1. Des troubles cognitifs : désorientation, discours confus, troubles de mémoire et un syndrome hallucinatoire progressif

2. Un syndrome anticholinergique : mydriase bilatérale persistante

3. Des troubles neurologiques : réflexes ostéo-tendineux diminués symétriquement et, peu après l'examen une crise convulsive cédant sous clonazépam.

Les examens neurologique et psychiatrique semblent écarter un syndrome épileptiforme et un début de schizophrénie. Une analyse toxicologique effectuée en urgence sur les urines, comprend la recherche de classes médicamenteuses (imipraminiques, benzodiazépines, et phénothiazines) et de stupéfiants (cannabis, cocaïne, opiacés et amphétamines).

Devant la persistance des signes cliniques anticholinergiques (mydriase, hallucinations) une recherche toxicologique spécifique dans les urines, a été demandée. ; kétamine (Ketalar ${ }^{\circledR}$ ), acide gamma-hydroxybutyrique, (Gamma-OH ${ }^{\circledR}$ ) et trihexyphenidyle (Artane ${ }^{\circledR}$ ).

Quelques heures après l'hospitalisation de la jeune fille, sa mère apporte un gobelet en plastique au fond duquel est pris en masse une poudre blanche humide, formant une pâte. Ce gobelet a été retrouvé dans la chambre de la jeune fille.

Les risques de rhabdomyolyse, liés à l'intoxication par la doxylamine conduisent à une surveillance étroite de l'activité créatine-phosphokinase (CPK) qui atteint $3800 \mathrm{UI} / \mathrm{L}$ (normales entre 15 et $130 \mathrm{UI} / \mathrm{L}$ ) 4 jours après l'intoxication puis diminue. On observe également une élévation de la troponine et une myoglobinurie. On n'observe pas de signe d'insuffisance rénale. La patiente sort de l'hôpital, 6 jours après son admission.

\section{Matériel et méthodes}

Une première série d'analyses immunochimiques est réalisée à la fois sur les urines et sur une solution aqueuse préparée par le laboratoire à partir de la poudre de nature inconnue, au moyen d'un automate COBAS MIRA PLUS ${ }^{\circledR}$, mettant en œuvre les réactifs Dade Berhing ${ }^{\circledR}$.

Une analyse chromatographique est mise en œuvre par CLHP couplée à une barrette de diodes (2). La méthodologie présente les caractéristiques suivantes.

\section{Extraction}

Les urines, ajustées à $\mathrm{pH}=5,0$, sont hydrolysées par environ $10 \mathrm{mg}$ de B-glucuronidase lyophilisée (Sigma $\left.{ }^{\circledR}\right)$. L'hydrolyse est effectuée pendant 12 heures à $45^{\circ} \mathrm{C}$. Après centrifugation, l'hydrolysat est traité par un tampon acétate d'ammonium $0,1 \mathrm{M}$, à $\mathrm{pH} 8$.

L'extraction solide-liquide se fait sur une colonne Isolute ${ }^{\circledR} \mathrm{HCX}$ (Bios Analytique ${ }^{\circledR}$ ) avant élution dans le système chromatographique.

La colonne est activée par $2 \mathrm{~mL}$ de méthanol (Carlo Erba $^{\circledR}$ ), rincée par $1 \mathrm{~mL}$ d'eau versable stérile (VER$S \mathrm{~L}^{\circledR}$ ), et équilibrée par $1 \mathrm{~mL}$ de tampon acétate d'ammonium. L'échantillon urinaire, ajusté à $\mathrm{pH}=8$, est passé sur la colonne. Les interférents sont élués par $1 \mathrm{~mL}$ d'eau, $1 \mathrm{~mL}$ d'acide chlorhydrique $0,01 \mathrm{M}$ puis $1 \mathrm{~mL}$ de méthanol.

Les analytes adsorbés sur la colonne sont élués par $2 \mathrm{~mL}$ d'un mélange de méthanol et d'ammoniaque $(98 / 2 ; \mathrm{v} / \mathrm{v})$. L'éluat est évaporé à sec sous azote et le résidu est repris par $200 \mu \mathrm{L}$ de phase aqueuse.

\section{Appareillage et conditions chromatogra- phiques}

La chaîne de chromatographie liquide est composée d'une pompe W600® Waters ${ }^{\circledR}$, d'un injecteur $717^{\circledR}$ Waters ${ }^{\circledR}$, d'un dégazeur et d'un détecteur $996^{\circledR}$ Waters ${ }^{\circledR}$ à barrettes de diodes. On utilise une colonne $\mathrm{C} 8$ Symmetry ${ }^{\circledR}$ de $250 \times 4.6 \mathrm{~mm}(5 \mu \mathrm{m})$, et une pré-colonne C8 20 x 4,6 mm $(5 \mu \mathrm{m})$ Waters $^{\circledR}$. Les données analytiques sont intégrées par un logiciel Millenium ${ }^{\circledR} 32$ Waters ${ }^{\circledR}$.

On utilise un gradient d'élution sur 25 min dont la composition initiale comprend $93 \%$ de phase aqueuse et $7 \%$ d'acétonitrile, et dont le débit est de $1 \mathrm{~mL} / \mathrm{min}$.

La phase aqueuse est un tampon phosphate $50 \mathrm{mM}$, ajusté à pH 3,8 [phosphate disodique, $\mathrm{Na}_{2} \mathrm{HPO}_{4}, \mathrm{H}_{2} \mathrm{O}$ 
$\left(\right.$ Merck $\left.^{\circledR}\right)$, eau stérile versable (VERSOL $\left.{ }^{\circledR}\right)$, acide chlorhydrique $1 \mathrm{~N}$ (Carlo Erba ${ }^{\circledR}$ )], filtrée (filtre Millipore ${ }^{\circledR}$ type $0,45 \mu \mathrm{m}$ ) et dégazée par ultrasons.

\section{Identification chromatographique}

Dans la bibliothèque $\operatorname{TOXICOL}^{\circledR}(3)$, qui référence plus de 850 molécules; la doxylamine a un temps de rétention de 11,1 minutes. Son spectre d'absorption U.V. présente deux maximums d'absorption à $200 \mathrm{~nm}$ et $260 \mathrm{~nm}$. La comparaison d'un spectre à analyser avec un spectre de la bibliothèque permet d'obtenir un «match angle». Cette valeur est recalculée en pourcentage d'homologie spectrale à partir duquel on peut affirmer l'identité d'un spectre (match angle $<10$ soit une homologie spectrale de $89 \%$ ).

L'analyse est effectuée à partir d'une solution aqueuse préparée au laboratoire d'une part à partir de la poudre blanche de nature inconnue, d'autre part sur les urines de la patiente après extraction et enfin sur une solution aqueuse de doxylamine de référence (succinate de doxylamine) préparée au laboratoire.

\section{Résultats}

Les résultats de l'analyse toxicologique de première intention sont tous négatifs (cannabis, cocaine, opiacés, benzodiazépines, barbituriques, imipraminiques et phénothiazines) aussi bien sur les urines que sur la solution aqueuse réalisée avec la poudre.

Les résultats de la recherche urinaire de kétamine (Kétalar ${ }^{\circledR}$ ), de gamma-hydroxybutyrate (Gamma-OH ${ }^{\circledR}$ ) et de trihexyphénidyle (Artane ${ }^{\circledR}$ ) sont tous négatifs.

L'analyse chromatographique de la solution aqueuse préparée à partir de la poudre blanche de nature inconnue montre un pic à $13,7 \mathrm{~min}$ de temps de rétention, avec un maximum d'absorption à 2,8 unités d'absorbance (figure 1). Son spectre d'absorption dans l'ultraviolet présente un maximum à deux longueurs d'ondes (200 nm et $260 \mathrm{~nm}$ ) (figure 3 ).

Par comparaison avec la bibliothèque spectrale, on obtient : homologie spectrale de $95 \%$ (match angle = 4,0 ) d'une part avec l'acide nicotinique dont le temps de rétention est 3,5 min, et une homologie spectrale de $91 \%$ (match angle $=8$ ) avec la doxylamine, dont le temps de rétention est de 13,7 min. La similitude spectrale observée entre le produit de nature inconnue et l'acide nicotinique et la doxylamine provient de la présence d'un noyau pyridine commun à ces molécules.

L'analyse des urines de la patiente montre également un pic à 13,7 min de temps de rétention, avec un spectre présentant deux pics aux longueurs d'onde : $190 \mathrm{~nm}$ et $300 \mathrm{~nm}$ (figure 2). Dans ce cas, l'homologie spectrale est de $96 \%$ (match angle $=3.5$ ) avec le spectre de la doxylamine.

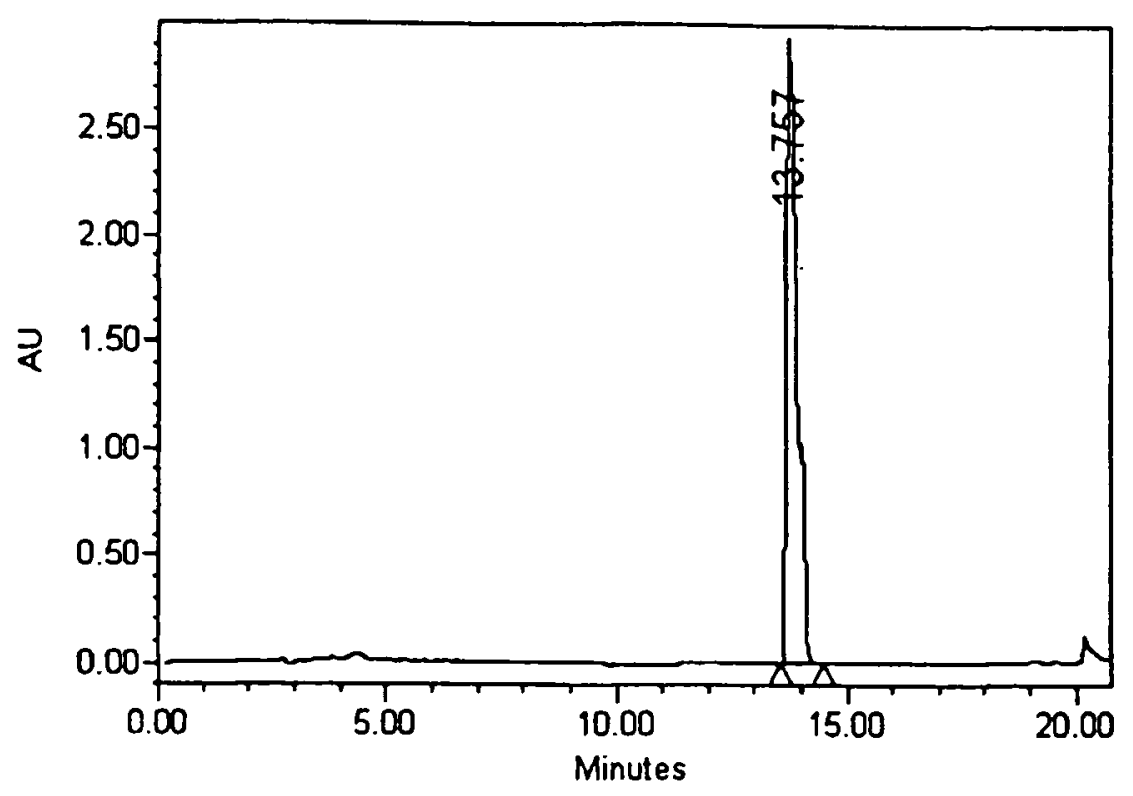

Figure 1 : Chromatogramme de la poudre.



Figure 2 : Chromatogramme des urines.

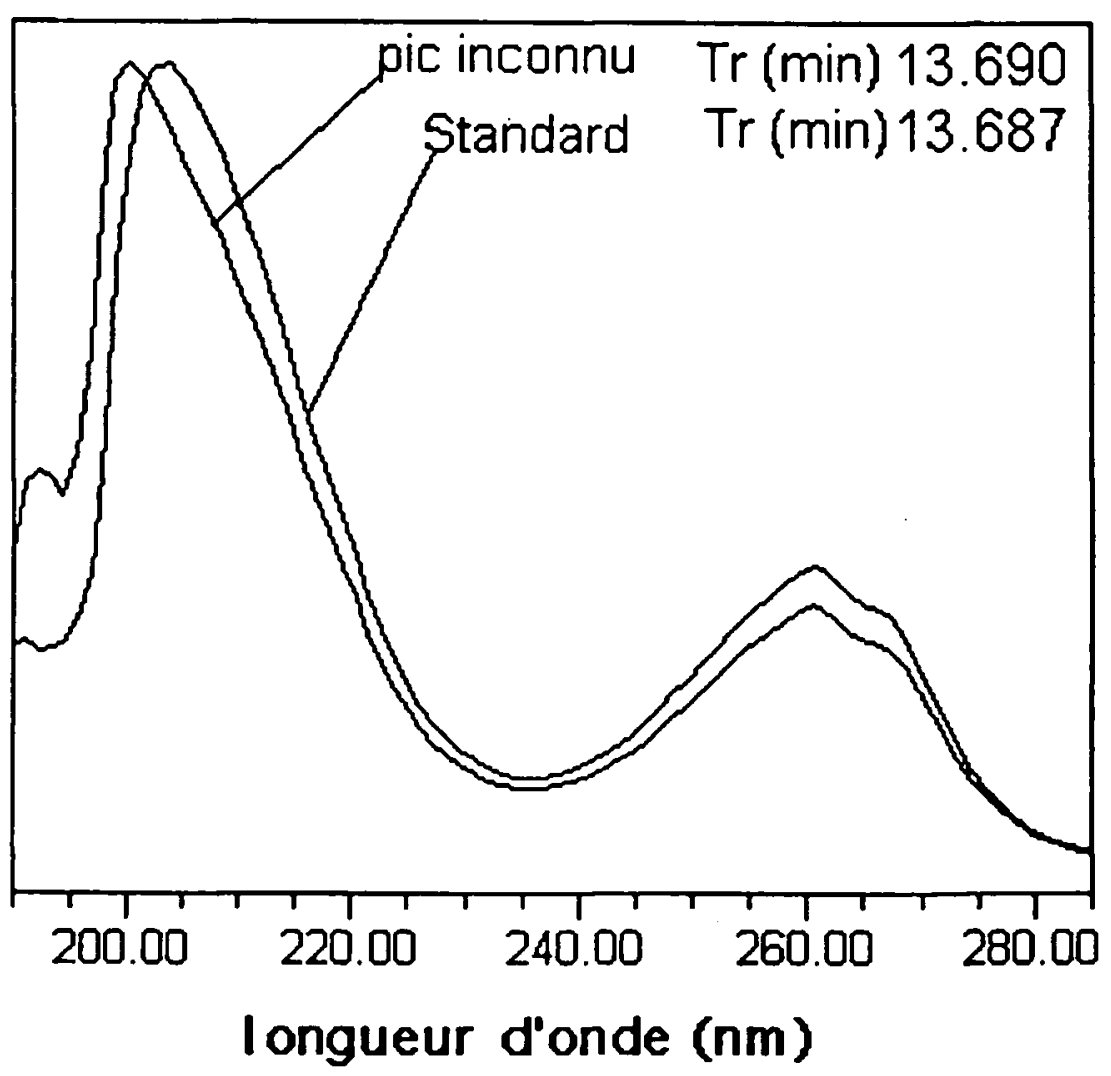

Figure 3 : Spectres de la doxylamine (standard et échantillon). 


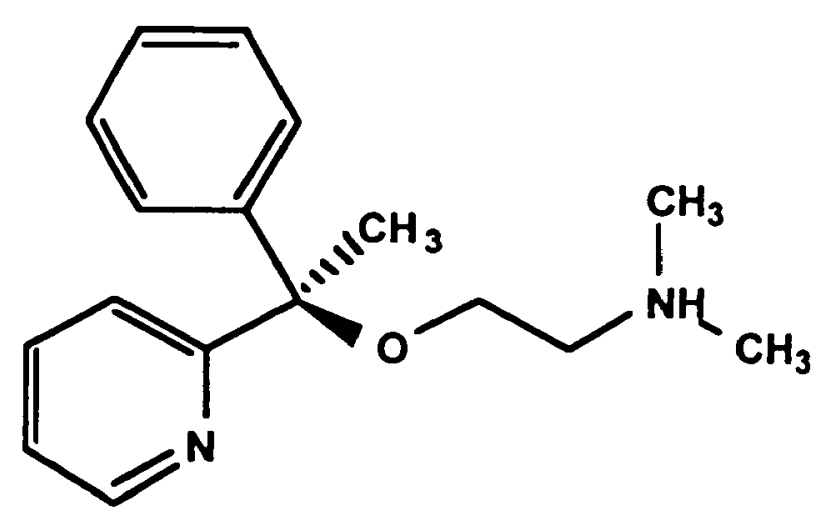

Figure 4 : Structure chimique de la doxylamine.

Le résultat ainsi obtenu lors de l'identification toxicologique au moyen d'une technique chromatographique (HPLC-BD), montre que la poudre analysée contient une grande quantité de doxylamine. Cette substance est également retrouvée en grande quantité dans les urines émises lors de l'admission $\dagger$.

Il n'a pas été réalisé de dosage sanguin.

La jeune fille avoue avoir acheté 5 boîtes de Donormyl ${ }^{\circledR}$ sans en expliquer la raison, et le psychiatre conclut à une intoxication médicamenteuse volontaire dans le contexte des difficultés de l'adolescence.

\section{Discussion}

La doxylamine est un médicament commercialisé sous le nom de Donormyl ${ }^{\circledR}$. Il s'agit d'un dérivé du diphénylméthane présentant des propriétés antihistaminiques H1. Sa structure est caractérisée par une chaîne éthanolamine. Elle possède des effets atropiniques et sédatifs qui sont mis à profit pour le traitement épisodique d'insomnies. On peut également utiliser la doxylamine en association avec un antitussif pour le soulagement de symptômes de type coryza ou sinusites inflammatoires.

Les intoxications à la doxylamine ont été décrites depuis longtemps dans la littérature et sont le plus souvent volontaires. Leur principale complication est la rhabdomyolyse (4) qui peut atteindre des niveaux extrêmement élevés (5) ; mais pendant la phase initiale de l'intoxication ce sont les signes anticholinergiques et cardiaques qui prédominent (6).

La pharmacocinétique de la doxylamine est caractérisée par un pic de concentration sanguine atteint au bout de deux heures pour le comprimé pelliculé, et une heure environ pour la forme effervescente. Dans le cas décrit ici, il s'agit d'une intoxication réalisée au moyen de la forme effervescente. La demi-vie plasmatique de la doxylamine est de l'ordre de 10 heures.

† Une analyse réalisée en parallèle à l'hôpital FernandWidal par chromatographie en phase gazeuse couplée à la spectrométrie de masse (CG/SM) indiquera la même résultat que celui qui a été déterminé à l'Hôtel-Dieu.
L'élimination, essentiellement rénale est réalisée, pour $60 \%$, sous forme inchangée. Il semble qu'en cas d'ingestion d'une dose élevée, voire en cas d'intoxication, l'élimination de la doxylamine est ralentie. C'est du moins ce qui a été démontré chez le singe (7).

L'élévation importante des CPK et la présence de myoglobine dans l'urine est évocatrice d'une rhabdomyolyse, même si celle-ci est restée discrète ou pauci-symptomatique. Elle a déjà été décrite lors d'intoxication par diverses substances toxiques ou médicamenteuses (8) et lors de comas prolongés fréquemment observés dans les d'intoxications par les substances psychotropes (benzodiazépines, barbituriques, phénothiazines) et la cocaïne. L'identification et le dosage de la doxylamine dans les milieux biologiques peuvent être effectués par une technique de chromatographie liquide (HPLC). Toutefois, la quantification des métabolites déméthylés, nordoxylamine et dinordoxylamine, et leurs conjugués N-acétylés (9) ou glucuronés (10) est plus habituellement réalisée par chromatographie en phase gazeuse couplée à la spectrométrie de masse (CPG/SM) ou par chromatographie sur couche mince (CCM) (11). Par ailleurs il a été démontré que le traitement de l'échantillon au moyen d'une hydrolyse en milieu acide, était susceptible de produire la formation $d$ 'artefacts analytiques (12).

\section{Conclusion}

Cette observation a permis de mettre en évidence une intoxication aigüe volontaire par la doxylamine (Donormyl $\left.{ }^{\circledR}\right)$. Ce médicament peut être actuellement dispensé en France en l'absence de prescription médicale. Il est utilisé à des fins sédatives, et présente d'importantes propriétés anticholinergiques. Son ingestion massive peut conduire à un tableau clinique grave nécessitant une hospitalisation de plusieurs jours. Cependant, l'étiologie précise reste difficile à déterminer et l'anamnèse auprès du patient peut s'avérer impossible. Seule l'analyse toxicologique de $2^{\text {eme }}$ intention, réalisée au moyen d'une méthode chromatographique couplée à un détecteur spécifique (HPLC-BD), permet de poser le diagnostic de certitude.

Dans le contexte actuel, où les drogues hallucinogènes à usage récréatif sont employées de façon croissante, il est donc important de rappeler le caractère potentiellement toxique de tous les psychotropes, même si le statut réglementaire peut ponctuellement leur conférer un caractère anodin.

Enfin, ce cas doit permettre de sensibiliser les pharmaciens d'officine lors de la vente de Donormyl ${ }^{\circledR}$, afin d'éviter un éventuel usage détourné, et le cas échéant en refuser la dispensation. 


\section{Références}

1. Lader M. Some adverse effects of antipsychotics : prevention and treatment. J. Clin. Psychiatry, $1999 ; 60$ Suppl. 12 : 18-21.

2. Herre S., Pragst F. Shift of the high-performance liquid chromatographic retention times of metabolites in relation to the original drug on an RP-8 column with acidic mobile phase. J. Chromatogr. B Biomed. Sci. Appl., $1997 ; 692: 111-26$.

3. Gaillard Y, Pépin G. Use of high-performance liquid chromatography with photodiode-array UV detection for the creation of a 600-compound library. Application to forensic toxicology. J Chromatogr A. 1997 ; 763 :149-63.

4. Leybishkis B., Fasseas P., Ryan K.F. Doxylamine overdose as a potential cause of rhabdomyolysis. Am. J. Med. Sci., 2001 ; 322 : 48-9.

5. Mendoza F.S., Atiba J.O., Krensky A.M., Scannell L.M. Rhabdomyolysis complicating doxylamine overdose. Clin. Pediatr. 1987 ; 26 : 595-7.

6. Frankel D., Dolgin J., Murray B.M. Non-traumatic rhabdomyolysis complicating antihistamine overdose. J. Toxicol. Clin. Toxicol. 1993 ; 31 : 493-6.

7. Slikker W.J.R., Holder C.L., Lipe G.W., Bailley J.R., Young J.F. Pharmacokinetics of doxylamine, a component of Bendectin, in the rhesus monkey. Reprod. Toxicol. $1989 ; 3$ : 187-96.
8. Hampel G., Horstkotte H., Rumpf K.W. Myoglobinuric renal failure due to drug-induced rhabdomyolysis. Hum. Toxicol., $1983 ; 2$ : 197-203.

9. Ganes D.A., Midha K.K. Identification in in vivo acetylation pathway for $\mathrm{N}$-dealkylated metabolites of doxylamine in humans. Xenobiotica, $1987 ; 17$ : 993-9.

10. Korfmarcher W.A., Holder C.L., Betowski L.D., Mitchum R.K. Identification of two glucuronide metabolites of doxylamine via thermospray / mass spectrometry and thermospray / mass spectrometry / mass spectrometry. J. Anal. Toxicol., $1987 ; 11: 182-4$.

11.Siek T.J., Dunn W.A. Documentation of a doxylamine overdose death: quantitation by standard addition and use of three instrumental techniques. J. Forensic Sci. 1993 ; $38: 713-20$.

12.Holder C.L., Korfmarcher W.A., Rushing L.G., Thompson H.C. Jr, Slikker W. Jr, Gosnell A.B. Formation of artifactual metabolites of doxylamine following acid hydrolysis. J. Chromatogr., 1987 ; 419: 113-22. 